\title{
Information coding via spontaneous oscillations in neural ensembles
}

\author{
Yuqing Wang and Z. D. Wang* \\ Department of Physics, The University of Hong Kong, Pokfulam Road, Hong Kong, People's Republic of China
}

(Received 14 October 1999; revised manuscript received 24 January 2000)

\begin{abstract}
How information is encoded and decoded via spontaneous oscillations is investigated by using an ensemble of Hodgkin-Huxley neurons. A signal can be encoded in spontaneous and highly irregular spike trains via high-order rate coding with the second-order statistics being relevant, in which the temporal structure and the correlation between the spikes are taken into account. Although the encoded information is implicitly contained in the spike train, it can be retrieved in the post-synaptic potential. The spontaneous oscillation is filtered and the irregularity of the spike train is suppressed. In particular, we show that an arbitrary signal can be transmitted reliably through spontaneous and highly irregular spike trains, and then be reconstructed downstream in the information transmission pathway.
\end{abstract}

PACS number(s): 87.16.Xa, 05.45.-a, 87.19.Dd

How information is encoded by neurons has been studied in the field of neuroscience for several decades [1-4]. Although it is generally accepted that neurons signal information through a sequence of the action potential, the neural code, by which information is transferred, remains elusive, and a debate on a noisy rate code and a precise temporal code has received renewed interest $[5,6]$. To understand the neural code, most effort has focused on the irregularity and the role of noise. However, the observed neural code exhibits also a feature of spontaneous oscillation or intrinsic bursting (see, e.g., the figure in Ref. [1]). In peripheral neural systems (PNS's), various sensory neurons output a mode of irregular intrinsic bursting when they are driven by a periodic input [7-9]. The post-gangalionic sympathetic nerves are composed of thousands of unmyelinated fibers. A large number of fibers fire the action potential at the same time to give bursts or waves of summed spikes. There have been substantial studies on how the bursting arises as well as how it affects signal transduction and neuroeffectors, but conclusions appear to be diverse (see Ref. [10] and references therein). In a central nervous system (CNS), neurons fire in highly irregular and complex spontaneous temporal patterns [11]. In recent years, a fast spontaneous oscillation (mainly $30-40 \mathrm{~Hz}$ ) has been observed in the brain cortex and was proposed to serve as a binding function $[12,13]$. Whether these spontaneous oscillation-dominated spike trains can carry and transmit signal, what kind of information is contained in the spike train, and how to retrieve it are yet to be answered. On the other hand, most experimental and theoretical studies are based on the spike trains, but the role of synapses has been paid little attention in information transmission. In fact, the spike should pass synapses to affect the neurons in the next stage of pathway, and what the neurons read is the post-synaptic potential (PSP). The synapse not only receives a membrane potential by the none-to-all law, but also changes the wave form of the spike train and enables a spatiotemporal integration of the spike train. How the synapse of the neuron, which will "read" the information en-

\footnotetext{
*Author to whom correspondence should be addressed. Electronic address: zwang@hkucc.hku.hk
}

coded by spike trains, affects signal transduction is quite interesting, but has been paid little attention.

In this paper, a high-order rate encoding and how to retrieve information via spontaneous oscillations are studied. The spontaneous oscillation is attributed to the excitability of the neuron. We show that, for these spontaneous oscillations, the pattern of the interspike interval (ISI) can be modulated by information. The information is encoded indirectly in the temporal patterns via the encoding by means of second-order statistics. This is a long-hypothesized representation of an information encoding scheme [14], which is now used to understand spontaneous and irregular spike trains. The synapse can be viewed as a filter or a decoding device. When the integration time of the synapse is of the same order of magnitude as the period of the spontaneous oscillation, the information encoded through the second-order statistics can be reconstructed in the PSP. The spontaneous oscillation and the irregularity of the spikes are filtered by the synapse. It seems that the spontaneous oscillation acts as a kind of carrier wave to enable a reliable information transmission without affecting the next neuron. The present scheme of encoding and decoding may be related to some experimental results.

We construct a two-layer summing neural cell ensemble by several hundreds of Hodgkin-Huxley (HH) neurons [15], as schematically shown in Fig. 1(a). The neurons in the first layer, where the information is encoded, are connected in parallel. Each neuron is subjected to a common input signal plus an independent noise source. The information is decoded in the second layer, where the spike trains are converged on the dendrites through excitatory synapses. The excitatory postsynaptic potential (EPSP) of these synapses is read by the neuron in the second layer. The $j$ th $\mathrm{HH}$ neuron is described by a set of four variables $(V, m, n, h)$ where $V$ is the membrane potential, $m$ and $h$ the activation and inactivation variables of sodium current, and $n$ the activation variable of potassium current. The corresponding equations read

$$
\begin{aligned}
\frac{d V}{d t}= & -g_{N a} m^{3} h\left(V-V_{N a}\right)-g_{K} n^{4}\left(V-V_{K}\right) \\
& -g_{L}\left(V-V_{L}\right)+I(t)+\eta,
\end{aligned}
$$



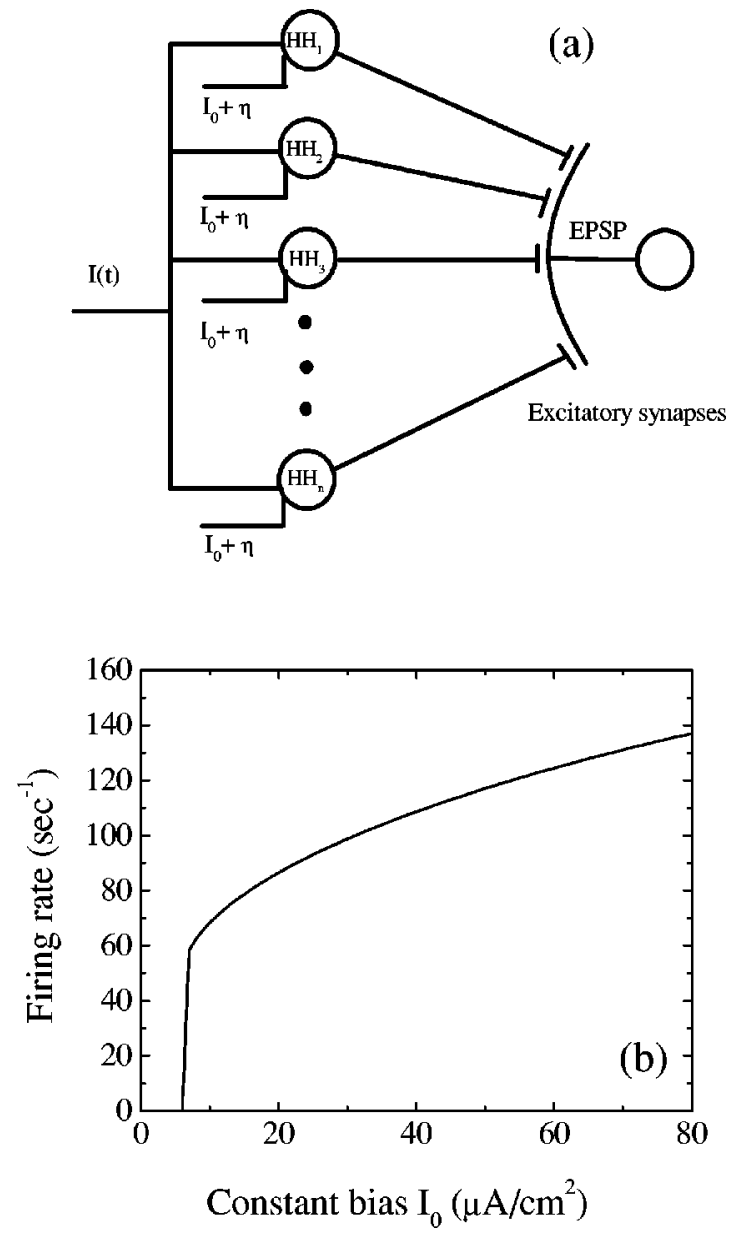

FIG. 1. (a) The schematic diagram of the ensemble of HodgkinHuxley neurons. (b) The firing rate versus the constant bias for a single $\mathrm{HH}$ neuron.

$$
\begin{gathered}
\frac{d m}{d t}=\frac{m_{\infty}(V)-m}{\tau_{m}(V)}, \\
\frac{d n}{d t}=\frac{n_{\infty}(V)-n}{\tau_{n}(V)}, \\
\frac{d h}{d t}=\frac{h_{\infty}(V)-m}{\tau_{h}(V)} .
\end{gathered}
$$

Here $I(t)$ is the input current. The independent noise $\eta$ is determined from an Ornstein-Uhlenbeck process $\tau_{c} d \eta / d t$ $=-\eta+\sqrt{2 D} \xi$, where $\xi$ is the Gaussaian white noise. $D$ and $\tau_{c}(=0.1 \mathrm{msec})$ are the intensity and correlation time of noise, respectively. The meanings and values of other parameters can be found in Ref. [16].

Due to a certain chemical transmission process at the synapse, a sharp spike induces the PSP with an $\alpha$ function $\left(t / \tau_{s}^{2}\right) \exp \left(-t / \tau_{s}\right)$ wave form [2]. The output spike train of each neuron in the first layer can be written as

$$
S_{j}(t)=\sum_{i} \delta\left(t-t_{i}\right)
$$
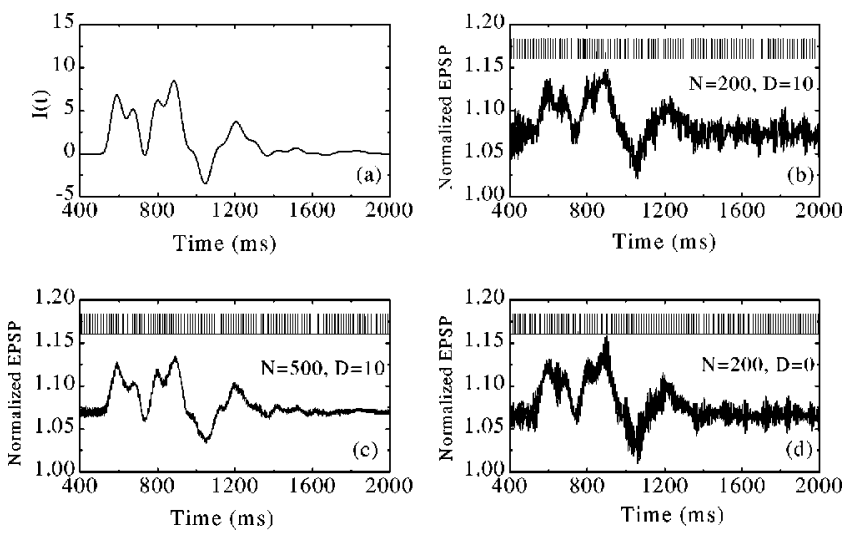

FIG. 2. The coding scheme for the aperiodic signal. (a) The input aperiodic signal. (b) The normalized EPSP for an ensemble of neurons with the number $N=200$ in the presence of noise ( $D$ =10). (c) $D=10$ and $N=500$. (d) $D=0$ (in the absence of noise) and $N=200$. Insets of $(\mathrm{b})-(\mathrm{d})$ : the corresponding encoded spike train of an arbitrarily chosen neuron in the ensemble. All neurons in the ensemble are subject to a randomly distributed constant bias $I_{0} \in[20,60]\left(\mu \mathrm{A} / \mathrm{cm}^{2}\right)$.

with firing times $t_{i}$ for the $n$th neuron. Then the total EPSP that the neuron in the second layer receives from the neural ensemble ( $N$ neurons) reads

$$
h^{s y n}=\sum_{j=1}^{N} J_{j} \int_{0}^{\infty}\left(s / \tau_{s}^{2}\right) \exp \left(-s / \tau_{s}\right) S_{j}(t-s) d s,
$$

where $\tau_{s}$ is the response rising time of the synapse and $J_{i}$ is the synaptic efficacy. Here, we assume that all the synapses are the same for simplicity and the axon transmission delay is neglected.

We wish to indicate first that, as an excitable oscillator, the spontaneous oscillations of $\mathrm{HH}$ neurons can be induced by imposing a large constant bias $I_{0}$. The neuron outputs periodic sequence of spikes for $I_{0}>6.2\left(\mu \mathrm{A} / \mathrm{cm}^{2}\right)$. The firing rate versus $I_{0}$ is plotted in Fig. 1(b). Because $I_{0}$ is independent of time, changing it is equivalent to changing the reversal potential of leakage channel $V_{L}$ in Eq. (1). From neurobiology, the spontaneous feature can be tuned by its own physiochemical adjustment [17], which can be modeled by changing the parameters of the equations. In the following simulation, we choose a large $I_{0}$ to keep the output spike train being dominated by spontaneous oscillations.

The scheme of coding for an aperiodic signal is demonstrated in Figs. 2(a)-2(d). As shown in Fig. 2(a), we choose an arbitrary aperiodic signal as the input signal. In an ensemble of 200 neurons, we first apply a random distributed constant bias $I_{0} \in[20,60]$ to each neuron. An independent noise with the same intensity $(D=10)$ is also applied to each neuron. After the transient period (500 $\mathrm{msec})$, the signal is put in. The spike train is irregular and spontaneous, which is shown in the inset of Fig. 2(b). The signal is reconstructed in the EPSP with high quality [Fig. 2(b)]; that is, what the neurons in the second layer read is almost the same as that the neurons in the first layer read. The quality of the retrieved signal is improved when the number of neurons in the ensemble increases [Fig. 2(c)]. In other words, the signal 


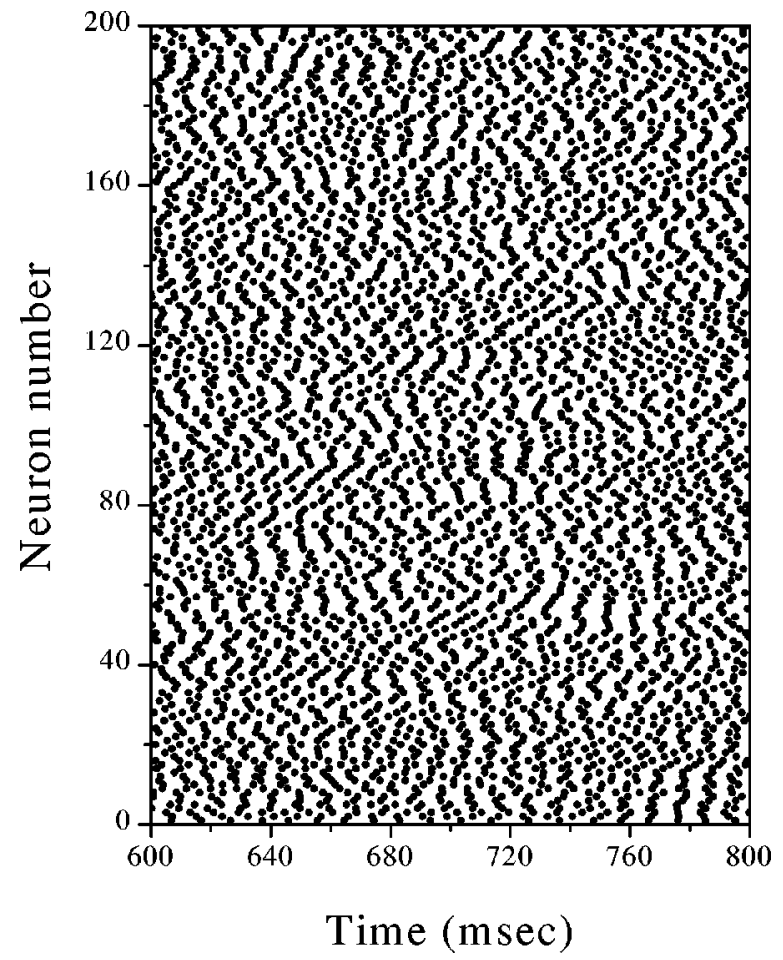

FIG. 3. The raster that records firing events of the ensemble in the case of Fig. 2(b), with a 200 msec window being chosen.

can be reliably transmitted downstream in the information transmission pathway even though in a very noisy environment.

In the absence of noise, the signal can also be reconstructed in the EPSP with high quality [Fig. 2(d)]. The spike train is only dominated by the spontaneous oscillation, as shown in the inset of Fig. 2(d), with the fluctuation in the EPSP coming from the uncertain firing times of spike train. Moreover, a signal transmission with good quality can be observed even when the size of ensemble is as small as 20 neurons (not shown here). The noise or large size of ensemble is unlikely the key prerequisite for understanding the coding scheme discussed here.
The raster that records the firing events of the system in the case of Fig. 2(b) is shown in Fig. 3. Because the constant bias is different for each neuron in the first layer, the firing rate varies from 87 to $125 \mathrm{sec}^{-1}$. It is seen that, not only is the firing rate of each neuron different, but also the firings are not synchronized. The spike trains are different even though the same signal is carried. On the other hand, for identical neurons and in the presence of noise, the same signal can be transmitted with the quality of the EPSP similar to Figs. 2(b)-2(d) (not shown here), but without synchronization among the spontaneous oscillation.

To demonstrate our coding scheme clearly, we choose a slow sine wave input current

$$
I(t)=I_{0}+I_{1} \sin (2 \pi f t) .
$$

For a given sine wave signal $\left(I_{1}=9 \mu \mathrm{A} / \mathrm{cm}^{2}\right.$ and $f$ $=5 \mathrm{~Hz})$, if we adjust the intensity of $I_{0}\left(=18 \mu \mathrm{A} / \mathrm{cm}^{2}\right)$, we can let the system experience spontaneous oscillations, which are shown in Fig. 4. In the absence of noise, the neuron bursts with a frequency $f_{s} \approx 80 \mathrm{~Hz}$, while the frequency of the input sine wave is $5 \mathrm{~Hz}$. This spontaneous $80 \mathrm{~Hz}$ oscillation is an intrinsic feature of the neuron due to its own excitability. If we plot the sequence of the ISI in Fig. 4(c), it is seen that the ISI varies between 10 and $16 \mathrm{msec}$ with a mean at about $13 \mathrm{msec}$, and the varying form is similar to the sine wave signal. This mode of temporal pattern is similar to the frequency modulation and the spontaneous bursting is the carrier wave. The ISI can be written as

$$
t_{i+1}-t_{i}=\Delta_{0}-\Delta_{1} \sin (2 \pi f t)
$$

with $\Delta_{0} \gg \Delta_{1}$.

In the presence of noise, the neuron experiences an irregular spontaneous bursting. As shown in Fig. 4(b), both the firing time and the ISI become random. From the usual analyses, such as the interspike interval histogram (ISIH) and fast Fourier transformation (FFT), we merely see spontaneous and irregular spike trains. However, if we plot the sequence of the ISI with different intensities of external noise in Fig. 4(d), we see that the ISI is still tuned by the sine
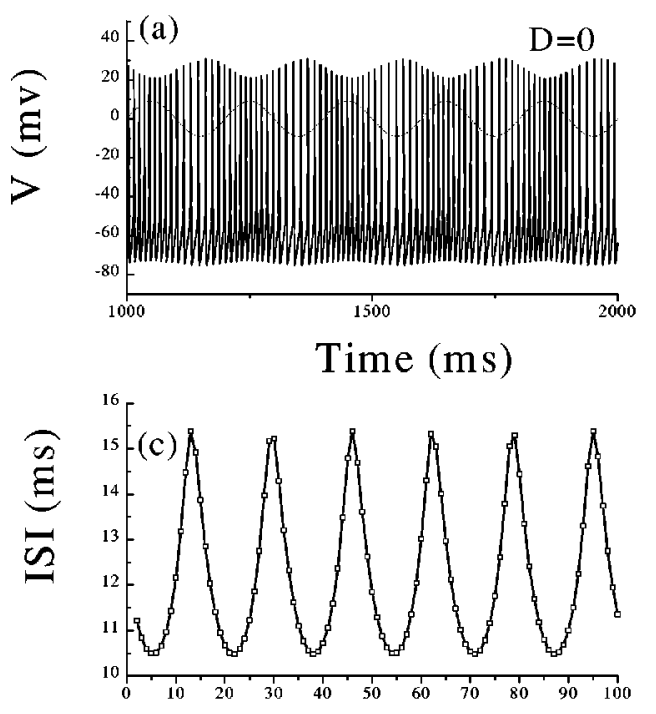

Spike Number
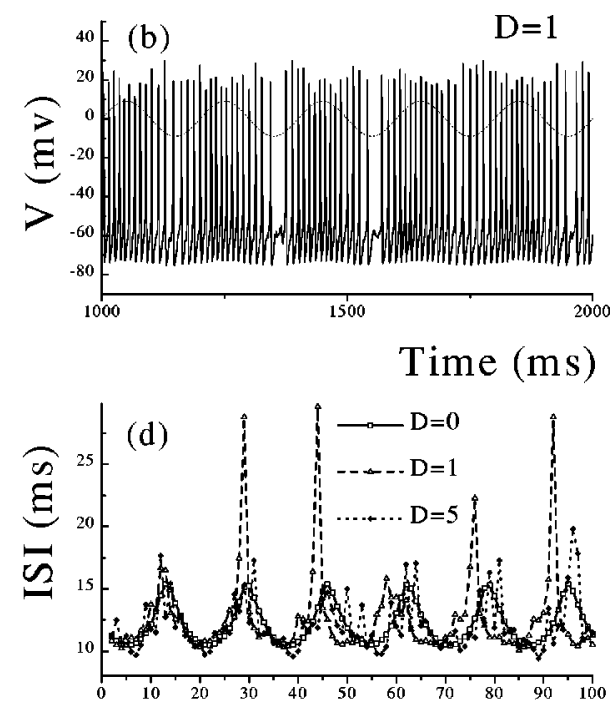

Spike Number
FIG. 4. The membrane potential against time for signal $I_{0}$ $=18 \mu \mathrm{A} / \mathrm{cm}^{2}, \quad I_{1}=9 \mu \mathrm{A} / \mathrm{cm}^{2}$, and $f=5 \mathrm{~Hz}$ with (a) $D=0$ and (b) $D=1$. The dashed lines are the input sine wave signal. (c) The corresponding interspike interval (ISI) vs spike number for (a). (d) The ISI vs spike numbers with different noise $(D=0, D=1, D$ $=5$, respectively) are plotted together. The counting of the spike number begins at $t=1000 \mathrm{msec}$. 
wave, although it is random. Note that the ISI can be described by a probability distribution

$$
P\left[t_{i+1} \mid t_{i}\right]=F(I(t)),
$$

which represents the probability of finding a spike at $t_{i+1}$ following the spike at $t_{i}$. According to Ref. [3], the usual firing rate would be determined by the probability of the firing at time $t_{i}$ [an exact definition is shown in Eq. (A.13) in Ref. [3]], while, in fact, Eq. (9) means that only the conditional probability $\mathrm{P}\left[\right.$ spike at time $t \mid$ spike at time $\left.t^{\prime}\right]$ is relevant in the present case. The firing rate is not given directly, but governed by the second-order statistics.

In fact, such a second-order rate coding is different from the usual rate coding in two aspects. First, for a single spike train, the usual firing rate means that the probability of firing at time $t_{i}$ is determined by the signal, without correlation among the spikes. So the usual stochastic-point-process description (the one mostly used is a homogeneous Poisson point process) can be introduced to analysze the spike train $[3,18]$, while in our situation the spike trains are highly correlated, with the correlation being determined by the spontaneous oscillation and modulated by the signal. As shown in Eq. (9), the conditional probability distribution does not depend solely on $\delta t=t-t^{\prime}$, which is different from the usual theoretical assumption [3]. Second, the role of an ensemble is different. For the usual rate coding, because the firing time is directly modulated by the signal, all spike trains in the ensemble resemble each other and seem to be synchronized to the external signal. As a result, it is reasonable to assume that the elements are identical, while in the present case, because the firing times are implicitly linked to the signal, no tendency of synchronization can be seen (Fig. 3). As long as the output spike trains are in such a phase that the spontaneous oscillation is dominant, the elements are intrinsically different. Note that the excitability of the neuron provides an additional degree of freedom for information coding, which gives much more flexibility for the information-coded spike trains, enabling the same signal transmitted through different patterns of spike trains.

The nonlinear feature of the present firing mode can be seen in Fig. 5. In the absence of noise [Fig. 5(a)], the peak of the ISIH is related to the spontaneous oscillation. From the return map of the ISI [the inset of Fig. 5(a)], we can observe the correlation between the consecutive spikes, while the exact timing of such a correlation is not favored. When the constant bias increases for $I_{0}>16.0\left(I_{1}=9.0\right.$ and $f$ $=5 \mathrm{~Hz}$ ), the system will be in such a quasi-periodic-state phase, with the firing patterns the same as that of Fig. 5 [19]. In the presence of noise, the peak of the ISIH is messed by noise, while the pattern of the return map is preserved to some extent.

For those spontaneous and highly irregular spike trains observed in the experiments, it is quite possible that there exists some kind of hidden high-order statistical feature, which leads to complexity in understanding such neural codes. For example, in an experiment on a motion-sensitive neuron in the fly's visual system [20], when the neurons were subjected to an aperiodic stimulus, the peristimulus time histogram (PSTH) indicates that the instantaneous firing rate was strongly modulated, but was not locked by the
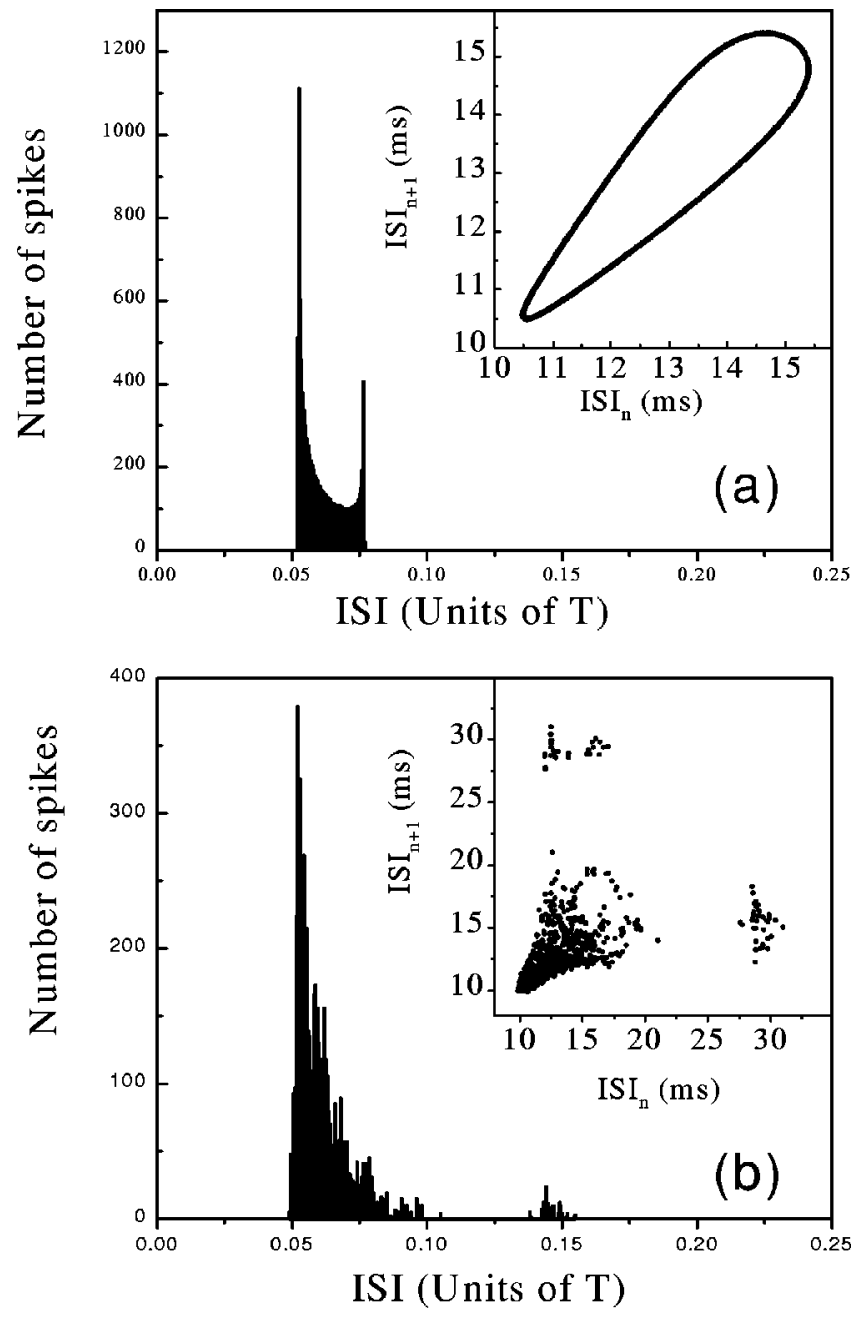

FIG. 5. $I_{0}=18 \mu \mathrm{A} / \mathrm{cm}^{2}, I_{1}=9 \mu \mathrm{A} / \mathrm{cm}^{2}$, and $f=5 \mathrm{~Hz}$. The interspike interval histogram (ISIH) when (a) $D=0$ and (b) $D$ $=1.0$. Insets of (a) and (b): the corresponding return map of the interspike interval.

stimulus. It is our understanding that the distribution or pattern of the ISI may be governed by high order statistics. In experiments on the periodically forced mechanoreceptor of macaque monkey [7] and cat retinal ganglion cell [8], one kind of firing mode is spontaneous bursting messed by noise, similar to Fig. 5(b). Because there is no trace of signal in the ISIH or PSTH, this spontaneous and highly irregular spike train was previously assumed to be irrelevant to information transmission [9]. Here we see the possibility that the signal can still be encoded. In the experiment of a neuron from area MT of monkey extrastriate visual cortex [5], the obtained instantaneous firing rate has a base mean firing rate and the rasters have shown that the firing is a kind of spontaneous oscillation pattern. It was argued that the base firing rate results from the random input of inhibitory and excitatory postsynaptic potential (IPSP and EPSP), which can be modeled as a random walk. Here we obtain the same result from a different mechanism that the excitability of the neuron itself can lead to a base mean firing rate. On the other hand, the pattern of instantaneous firing rate is complicated and irregular, possibly due to the correlation between the spikes and/or the irregularity of the spike trains.

The synapse can decode the signal carried by the second- 
(a)

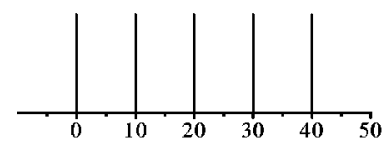

(b)
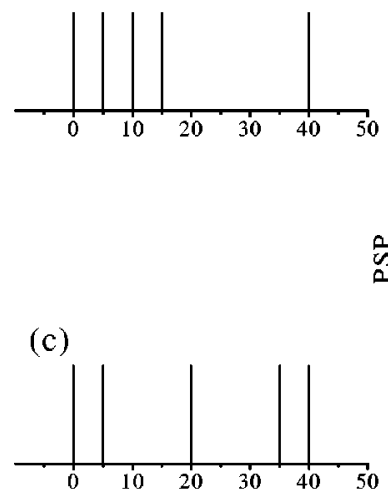

Time (ms)

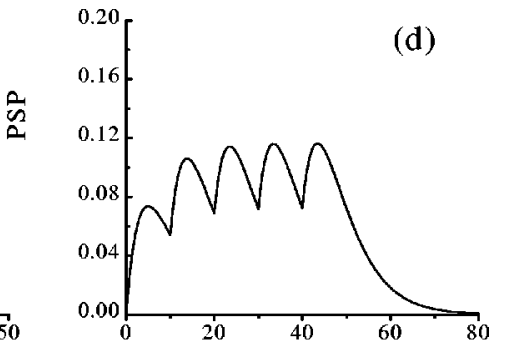

(e)

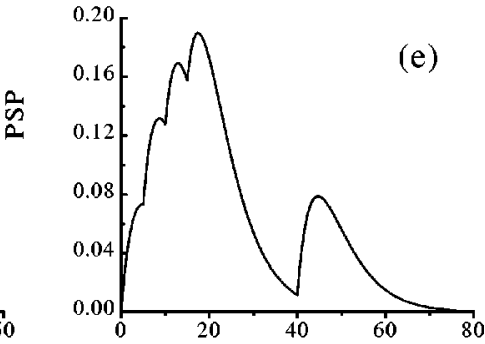

(f)
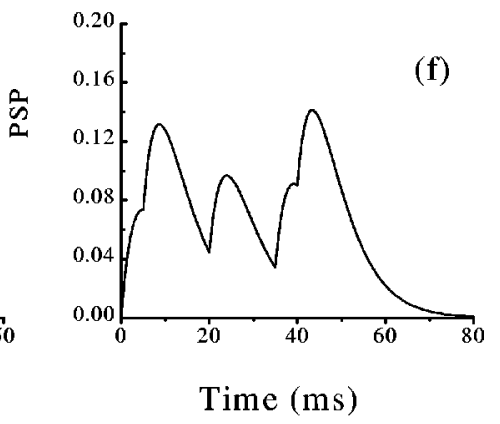

FIG. 6. Three different firing patterns [(a)-(c)] and their corresponding PSP [(b)-(f)]. The mean firing rate is $100 \mathrm{spike/sec,} \mathrm{but}$ the ISI patterns are different. The PSP is calculated by taking $\tau_{s}$ $=5 \mathrm{msec}$.

order statistics if the integration time of the synapses is in the same order magnitude as the average period of the spontaneous oscillation. Theoretically, as shown in Eq. (6), the resulted EPSP includes the effects of all the previous spikes. Because the $\alpha$ function decays rapidly, the temporal integration can be considered to be the summing of spikes in a given period of time. The time window that the $\alpha$ function decays to $1 / e$ of its maximum value is referred to as the integration time of the synapse, which is determined by $\tau_{s}$. The synapse can convert the ISI pattern to the the PSP, when the ISI is within the integration time of the synapse. As shown in Figs. 6(a)-6(c), three spike train patterns have the same mean firing rate (five spikes in $50 \mathrm{~ms}$ time window), but with different ISI patterns. We choose the integration time to be $16 \mathrm{msec}\left(\tau_{s}=5 \mathrm{msec}\right)$. The resulted PSPs tend to overlap for consecutive spikes. Different temporal patterns of the ISI will give different amplitudes of the PSP [see Figs. 6(d)-6(f)]. We note that a series of experiments on Aplysia done about 30 years ago [10] showed that two different patterns of presynaptic pulses, with the same mean frequency but different second-order statistics, give different postsynaptic responses. We may understand now that the reason is due to the wave form of the PSP and the integration time of the synapse.

For the spontaneous-oscillation-carried sine wave signal, we first look into the integration of only one synapse. In the absence of noise, the resulted EPSP is shown in Fig. 7(a). Here we choose $\tau_{s}=5 \mathrm{msec}$, and the mean ISI of bursting is
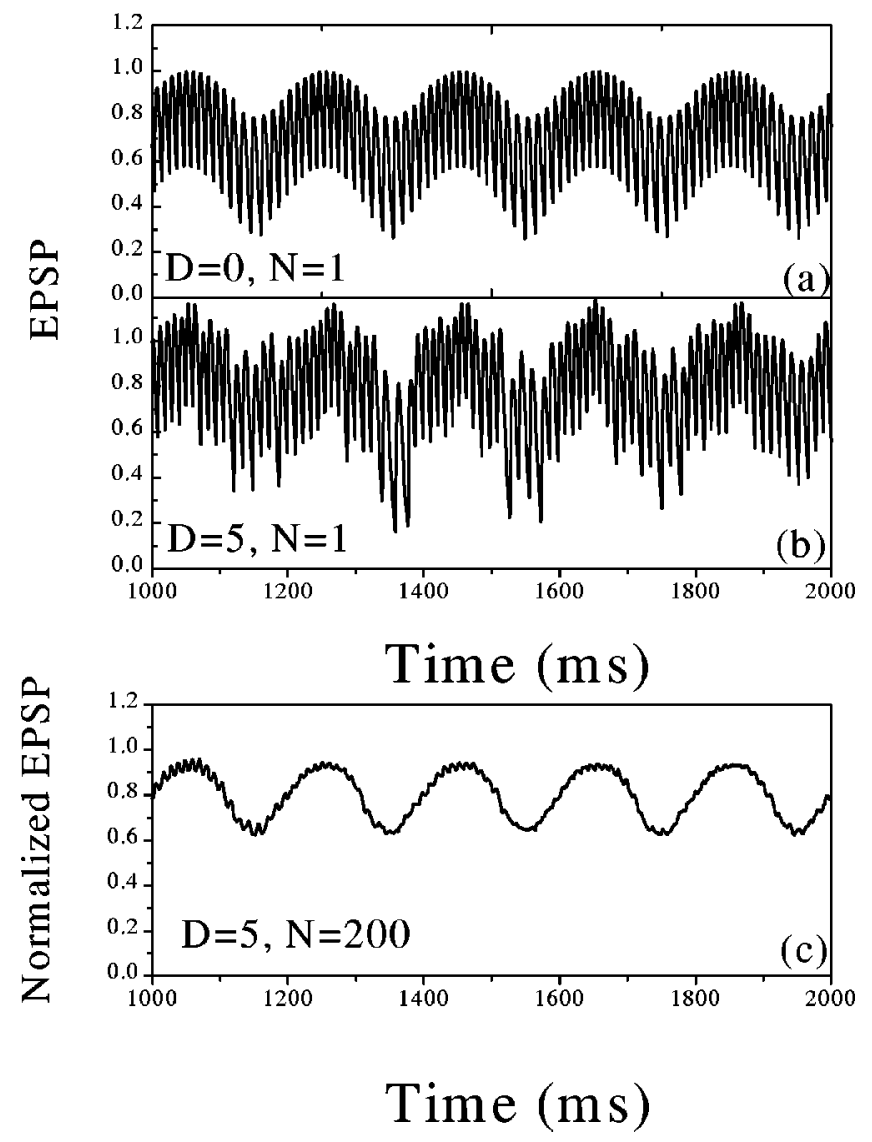

FIG. 7. The excitatory postsynaptic potential (EPSP) with one spike train exerting on the synapse vs time for $\tau_{s}=5 \mathrm{msec}$ with (a) $D=0$ and (b) $D=5$. (c) The normalized EPSP with 200 noiseindependent spike trains exerting on the synapses vs time for $\tau$ $=5 \mathrm{msec}$ and $D=5$. The input spike train is the output of the $\mathrm{HH}$ neuron with input current parameters $I_{0}=18 \mu \mathrm{A} / \mathrm{cm}^{2}, I_{1}$ $=9 \mu \mathrm{A} / \mathrm{cm}^{2}$ and $f=5 \mathrm{~Hz}$. When the external noise is switched off, the oscillation of the membrane potential is shown in Fig. 4(b).

about 13 msec [see Fig. 4(d)]. Through the temporal integration of spike trains, the sine wave can be retrieved and is explicitly demonstrated in the EPSP. When a strong external noise is applied to the system, as shown in Fig. 7(b), the EPSP is messed, indicating that only the temporal integration of one synapse is unable to retrieve the signal reliably from the highly irregular spike trains. However, the result is quite different if we consider an ensemble. The normalized EPSP for an ensemble with 200 neurons $\left(h^{\text {syn }} / 200\right)$ is plotted in Fig. 7(c). It is shown that, when both the temporal and spatial integration are taken into account, the signal is reconstructed with very high quality. We have examined the cases with various intensities and frequencies of the signal. As we can always tune the constant bias $I_{0}$ to ensure the output spike train to be dominated by spontaneous oscillations and choose an appropriate integration time, the basically same phenomenon is observed (not shown here). That is, as long as the pattern of output spike train falls in such a quasiperiodic regime, where the spontaneous oscillation can serve as the carrier wave, information can be encoded implicitly via the high-order statistics.

It is worth pointing out that, the input signal is very large in the present case, being essentially different from the sto- 
chastic resonance where the signal is weak $[21,22]$. Here the noise messes the spike train in signal transduction, while, on the other hand, the independent local noise randomizes the phases of spontaneous oscillations in the ensemble, enabling the synapses to filter these spontaneous oscillations through spatiotemporal integration, as shown in Fig 7(c).

The present coding scheme may provide a valuable understanding of the activity of the sympathetic nerves. First, the bursting comes from the spontaneous oscillation of the neuron due to its own excitability, and encodes signal as the carrier wave. This mechanism favors the model of Fig. 5(d) in Ref. [10], which it was proposed to describe how baroreceptor signals interact with sympathetic drive to cause grouping of burst discharges as well as to affect the overall burst probability. On the other hand, the sympathetic neuroeffector delay has usually a much longer time than the period of the bursting [23]. So the spontaneous oscillation is filtered, as shown in Fig. 6(d). Although spontaneous oscillations have nothing to do with the effector response, they are important in signal transduction.

Finally, we wish to make a remark on the data processing of neural experiments. In many experiments, different definitions of the firing rate appear to be confusing. Some are simply the conventional definition [24], while others are related to the usual signal processing method by introducing symmetric Hanning window [25] or various specifically defined windows $[20,26]$. The sizes of these windows chosen by different experimentalists vary significantly. In fact, these methods are a kind of temporal integration. The PSTH obtained by these methods reflects only partially the effect of the synapses. We now learn that the size of the window should be chosen seriously and carefully. It should be determined by the the synapse of the neuron which will "read" the spike trains. For example, in the CNS, $2 \leqslant \tau_{s} \leqslant 5 \mathrm{msec}$ is realistic [2], which corresponds to $6-16 \mathrm{msec}$ integration time of the synapse. This size of window is likely biologically related. In our opinion, a window of $\alpha$ function with appropriate size is likely more reasonable for the data processing in neural experiments.

In summary, we have studied information coding via spontaneous oscillations in an ensemble of $\mathrm{HH}$ neurons. We have found that a signal can be encoded implicitly in spontaneous and highly irregular spike trains via high-order rate coding with the second-order statistics being relevant. The signal is reconstructed in the PSP through the spatiotemporal integration of the synapses. We have shown that an arbitrary signal can be transmitted reliably downstream in the information transmission pathway, by making use of spontaneous oscillations in a noisy environment.
[1] D. Ferster and N. Spruston, Science 270, 756 (1995).

[2] W. Gerstner and J.L. van Hemmen, in Models of Neural Networks II, edited by E. Domany, J.L. van Hemmen, and K. Schulten (Springer-Verlag, Berlin, 1994), pp. 1-85.

[3] F. Rieke, D. Warland, R.R. de Ruyter van Steveninck, and W. Bialek, Spikes: Exploring the Neural Code (MIT Press, Cambridge, MA, 1997).

[4] N. Brenner, O. Agam, W. Bialek, R.R. de Ruyter van Steveninck, Phys. Rev. Lett. 81, 4000 (1998); S.P. Strong, R. Koberle, and R.R. de Ruyter van Steveninck, and W. Bialek, ibid. 80, 197 (1998).

[5] M.N. Shadlen and W.T. Newsome, Curr. Opin. Neurobiol. 4, 569 (1994).

[6] W.R. Softky, Curr. Opin. Neurobiol. 5, 239 (1995).

[7] W.H. Talbot, I. Darian-Smith, H.H. Kornhuber, and V.B. Mountcastle, J. Neurophysiol. 31, 301 (1968).

[8] T. Ogawa, P.O. Bishop, and W.R. Levick, J. Neurophysiol. 6, 2 (1966).

[9] D.R.F. Irvine, in Progress in Sensory Physiology 7: The Auditory Brainstem, edited by H. Autrum, D.O. Hoson, E.R. Perl, R.F. Schmidt, and W.D. Willis (Springer-Verlag, Berlin, 1986), Chap. 4.

[10] R.M. McAllen and S.C. Mapas, Clin. Exp. Pharmacol. Physiol. 24, 791 (1997).

[11] M. Steriade, I. Timofeev, N. Dürmüller, and F. Grenier, J. Neurophysiol. 79, 483 (1998).

[12] R. Llinás and U. Ribary, Proc. Natl. Acad. Sci. USA 88, 2078 (1993).

[13] W. Singer and C.M. Gray, Annu. Rev. Neurosci. 18, 555 (1995).

[14] J.P. Segundo and A.M. Simmons, J. Exp. Biol. 40, 643 (1963).
[15] X. Pei, L. Wilkens, and F. Moss, Phys. Rev. Lett. 77, 4679 (1996).

[16] A.L. Hodgkin and A.F. Huxley, J. Physiol. (London) 117, 500 (1952); D. Hansel, G. Mato, and C. Meunier, Phys. Rev. E 48, 3470 (1993).

[17] I.B. Levitan and L.K. Kaczmarek, The Neuron: Cell and Molecular Biology (Oxford University Press, New York, 1991).

[18] H.C. Tuckwell, Stochastic Processes in the Neurosciences (Society for Industrial and Applied Mathematics, Philadelphia, 1989).

[19] Y. Wang, Z.D. Wang, and W. Wang, J. Phys. Soc. Jpn. 69, 276 (2000).

[20] R.R. de Ruyter van Stevenick, G.D. Lewen, S.P. Strong, R. Koberle, and W. Bialek, Science 275, 1805 (1997).

[21] J.J. Collins, C.C. Chow, and T.T. Imhoff, Nature (London) 378, 362 (1995); D.R. Chialvo, A. Longtin, and J. MllerGerking, Phys. Rev. E 55, 1798 (1997); A. Neiman, L. Schimansky-Geier, and F. Moss, ibid. 56, R9 (1997).

[22] W. Wang and Z.D. Wang, Phys. Rev. E 55, 7379 (1997); W. Wang, G. Chen, and Z.D. Wang, ibid. 56, 3728 (1997); W. Wang, Y. Wang, and Z.D. Wang, ibid. 57, R2527 (1998); Y. Wang, D.T.W. Chik, and Z.D. Wang, ibid. 61, 740 (2000); Y. Wang, Z.D. Wang, and W. Wang, J. Phys. Soc. Jpn. 67, 3637 (1998).

[23] H.R. Warner and A.A. Cox, J. Appl. Physiol. 17, 349 (1962); M. Kunimoto, K. Kirno, E. Elam, and B.G. Wallin, J. Physiol. (London) 442, 391 (1991).

[24] E.D. Adrian and Y. Zotterman, J. Physiol. (London) 61, 465 (1926).

[25] J.J. Collins, T.T. Imhoff, and P. Grigg, J. Neurophysiol. 76, 642 (1996).

[26] K. Macleod, A. Bäcker, and G. Laurent, Nature (London) 395, 693 (1998). 